\title{
THE CONSERVATION RELATION FOR CUSPIDAL REPRESENTATIONS
}

\author{
by
}

Alberto Mínguez

\begin{abstract}
Let $\pi \in \operatorname{Cusp}\left(\mathrm{U}\left(V_{n}\right)\right)$ be a smooth cuspidal irreducible representation of a unitary group $\mathrm{U}\left(V_{n}\right)$ of dimension $n$ over a non-Archimedean locally compact field. Let $W_{m}^{ \pm}$the two isomorphism classes of Hermitian spaces of dimension $m$, and the denote by $\tau^{+} \in \operatorname{Cusp}\left(\mathrm{U}\left(W_{m^{+}}^{+}\right)\right)$and $\tau^{-} \in \operatorname{Cusp}\left(\mathrm{U}\left(W_{m^{-}}^{-}\right)\right)$the first non-zero theta lifts of $\pi$. In this article we prove that $m^{+}+m^{-}=2 n+2$, which was conjectured in [HKS, Speculations 7.5 and 7.6]. We prove similar equalities for the other dual pairs of type I : the symplecticorthogonal dual pairs and the quaternionic dual pairs.
\end{abstract}

\section{Introduction}

Let $\mathrm{F}$ be a non-Archimedean locally compact field, of characteristic not equal to 2 , and fix $\psi: \mathrm{F} \rightarrow \mathbb{C}^{\times}$a non-trivial additive character of $\mathrm{F}$.

Let $\left(G, G^{\prime}\right)$ be an irreducible dual pair of type I. Dual pairs of type I come in three types:

(I) Let $\mathrm{E}$ be a quadratic extension of $\mathrm{F}$ and $V_{n}$ and $W_{m}$ some E-vector spaces of dimension $n$ and $m$ equipped, respectively, with a non-degenerate Hermitian and a nondegenerate skew-Hermitian form. Then $\mathrm{G}=\mathrm{U}\left(V_{n}\right), \mathrm{G}^{\prime}=\mathrm{U}\left(W_{m}\right)$ are unitary groups defined over F.

2000 Mathematics Subject Classification. - 11F27, 22E50.

Key words and phrases. - Theta correspondence; theta dichotomy; representations of p-adic groups.

Partially supported by EPSRC grant EP/G001480/1, MTM2007-66929 and FEDER. 
(II) Let $V_{n}$ and $W_{m}$ be some F-vector spaces of dimension $n$ and $m$ equipped, respectively, with a quadratic and a symplectic form. Then $\mathrm{G}=\mathrm{O}\left(V_{n}\right)$ is an orthogonal group and $\mathrm{G}^{\prime}=\operatorname{Sp}\left(W_{m}\right)$ is a symplectic group defined over $\mathrm{F}$.

(III) Let $\mathrm{D}$ be the quaternion F-algebra and $V_{n}$ and $W_{m}$, some D-vector spaces of dimension $n$ and $m$ equipped, respectively, with a non-degenerate Hermitian and a nondegenerate skew-Hermitian form. Then $\mathrm{G}=\mathrm{U}\left(V_{n}\right), \mathrm{G}^{\prime}=\mathrm{U}\left(W_{m}\right)$ are quaternionic unitary groups defined over F.

We will give more details of each of these dual pairs in the forthcoming sections.

Denote by $\widetilde{\mathrm{G}}$ and $\widetilde{\mathrm{G}^{\prime}}$ their pre-images in the metaplectic group. The metaplectic group is equipped with a complex representation, canonically attached to $\psi$, the Weil representation, also called the metaplectic representation, which will be denoted in this introduction by $\omega$. We are interested in its restriction to the product $\widetilde{\mathrm{G}} \times \widetilde{\mathrm{G}^{\prime}}$. We say that a genuine irreducible representation $\pi$ of $\widetilde{G}$ occurs in the theta correspondence for the dual pair $\left(\mathrm{G}, \mathrm{G}^{\prime}\right)$ if $\pi$ occurs as a quotient of $\omega$. The maximal quotient of $\omega$ which is $\pi$-isotypic has the form $\pi \otimes \Theta\left(\pi, W_{m}\right)$, where $\Theta\left(\pi, W_{m}\right)$ is a smooth representation of $\widetilde{\mathrm{G}^{\prime}}$ [MVW, 2.III.5]. The Howe duality principle asserts that $\Theta\left(\pi, W_{m}\right)$ has a unique irreducible quotient, denoted by $\theta\left(\pi, W_{m}\right)$. This principle is known to hold if the residue characteristic of $\mathrm{F}$ is not 2 [Wa1] or when $\pi$ is a cuspidal representation [MVW, page 69]. In this article, we will just be interested in cuspidal representations so we will not need to suppose that the residue characteristic of $\mathrm{F}$ is different from 2 . We will write $\theta\left(\pi, W_{m}\right)=0$ if $\pi$ does not occur in the theta correspondence for $\left(\widetilde{\mathrm{G}}, \widetilde{\mathrm{G}^{\prime}}\right)$. One of the main problems in the theory of the theta correspondence is to determine whether $\theta\left(\pi, W_{m}\right)$ is 0 or not.

Fix the integer $n$ and a genuine irreducible representation $\pi$ of $\widetilde{G}$. Consider the integer $m$ as a variable. Theta conservation principle predicts the first occurrence of $\pi$ in the theta correspondence, that is, the smallest integer $m$ such that $\theta\left(\pi, W_{m}\right)$ is not zero. This conjecture was first stated in $[\mathbf{H K S}]$ and extended to the symplectic-orthogonal dual pairs in $[\mathbf{K R}]$, where the conjecture was proved for many irreducible representations (including cuspidal representations). In [GTn], the conjecture was made for quaternionic dual pairs. It has been shown to be of great interest for proving that a theta lift is non-zero (see, for example, $[\mathbf{G T k}]$ for a beautiful application).

The precise statement of this conjecture depends on the dual pair and our simple argument applies only when $\pi$ is a cuspidal representation (in this case Howe duality principle holds independently of the residual characteristic of $\mathrm{F}$ so we will not need to suppose that it is different from 2). We will give the technical details in the forthcoming 
sections. Section 1 is devoted to the case where $G, G^{\prime}$ are two unitary groups, Section 2 deals with symplectic-orthogonal dual pairs and finally, in Section 3, we will treat the case of quaternionic dual pairs.

\section{The dual pair $(\mathrm{U}(n), \mathrm{U}(m))$}

Let $\mathrm{E}$ be a quadratic extension of $\mathrm{F}$ with associated quadratic character $\epsilon_{\mathrm{E} / \mathrm{F}}$. Let $V_{n}$ and $W_{m}$ be some E-vector spaces of dimension $n$ and $m$ equipped, respectively, with a non-degenerate skew-Hermitian and a non-degenerate Hermitian form. Then the isometry groups $\mathrm{U}\left(V_{n}\right)$ and $\mathrm{U}\left(W_{m}\right)$ of the spaces $V_{n}$ and $W_{m}$ form a reducive dual pair in $\operatorname{Sp}\left(V_{n} \otimes\right.$ $\left.W_{m}\right)$. This dual pair is split : attached to a fixed pair of characters $\chi=\left(\chi_{n}, \chi_{m}\right)$ of $\mathrm{E}^{\times}$ with

$$
\left.\chi_{m}\right|_{\mathrm{F}^{\times}}=\epsilon_{\mathrm{E} / \mathrm{F}}^{m} \text { and }\left.\chi_{n}\right|_{\mathrm{F}^{\times}}=\epsilon_{E / \mathrm{F}}^{n}
$$

there are splitting homomorphisms

$$
\begin{aligned}
i_{\chi_{m}}: \mathrm{U}\left(V_{n}\right) & \rightarrow \widetilde{\operatorname{Sp}}\left(V_{n} \otimes W_{m}\right) \\
i_{\chi_{n}}: \mathrm{U}\left(W_{m}\right) & \rightarrow \widetilde{\operatorname{Sp}}\left(V_{n} \otimes W_{m}\right) .
\end{aligned}
$$

Using these splittings, we consider the Weil representation restricted to $\mathrm{U}\left(V_{n}\right) \times \mathrm{U}\left(W_{m}\right)$, and, for a cuspidal representation $\pi$ of $\mathrm{U}\left(V_{n}\right)$, denote by $\theta_{\chi}\left(\pi, W_{m}\right)$ the theta lift of $\pi$ to $\mathrm{U}\left(W_{m}\right)$.

For a fixed dimension $m$, there are precisely two isomorphism classes of Hermitian spaces, $W_{m}^{ \pm}$, so that

$$
\epsilon_{\mathrm{E} / \mathrm{F}}\left((-1)^{\frac{m(m-1)}{2}} \operatorname{det} W_{m}^{ \pm}\right)= \pm 1,
$$

and we consider the collection of representations $\theta_{\chi}\left(\pi, W_{m}^{ \pm}\right)$as $m$ and \pm vary. Fix a parity of $m$ and define $m^{+}$(resp. $m^{-}$) to be the smallest integer $m$ with that fixed parity such that $\theta_{\chi}\left(\pi, W_{m}^{+}\right) \neq 0\left(\operatorname{resp} . \theta_{\chi}\left(\pi, W_{m}^{-}\right) \neq 0\right)$.

By [HKS, Corollaries 4.4], one has that

$$
\max \left\{m^{+}, m^{-}\right\} \geq n+1
$$

and by [HKS, Corollaries 4.5], one also knows that

$$
\min \left\{m^{+}, m^{-}\right\} \leq n+1,
$$

Theorem 1.1. - We have

$$
m^{+}+m^{-}=2 n+2 \text {. }
$$


This equality was conjectured in [HKS, speculations 7.5 and 7.6] to hold for every irreducible representation $\pi$. Following the ideas in $[\mathbf{K R}]$, the conjecture has been proved independently in $[\mathbf{G G}]$ with the same restrictions as in $[\mathbf{K R}]$.

Proof. - Define the skew-Hermitian space $V_{n+2}=V_{n} \oplus H$, where $H$ is a hyperbolic space. Denote by $P_{1}$ the parabolic subgroup of $\mathrm{U}\left(V_{n+2}\right)$ associated to this decomposition. We will write $\tau^{+}=\theta_{\chi}\left(\pi, W_{m^{+}}^{+}\right)$and $\tau^{-}=\theta_{\chi}\left(\pi, W_{m^{-}}^{-}\right)$. It is known that $\tau^{ \pm}$is a cuspidal representation of $\mathrm{U}\left(W_{m^{ \pm}}^{ \pm}\right)$.

Consider the theta lift $\theta_{\chi}\left(\tau^{+}, V_{n+2}\right)$. By $[\mathbf{M V W}$, page 69], the Jacquet functor $J_{P_{1}}\left(\theta_{\chi}\left(\tau^{+}, V_{n+2}\right)\right)$ is irreducible and by Ibid or [Han, Theorem 3.1], $\theta_{\chi}\left(\tau^{+}, V_{n+2}\right)$ is a subquotient of the parabolically induced representation $\operatorname{Ind}_{P_{1}}^{\mathrm{U}\left(V_{n+2}\right)}\left(||^{\left(m^{+}-n-1\right) / 2} \chi_{m} \otimes \pi\right)$. As the Jacquet functor of this induced representation, by the geometric lemma of $[\mathbf{B Z}$, 2.12], is reducible we deduce that $\operatorname{Ind}_{P_{1}}^{\mathrm{U}\left(V_{n+2}\right)}\left(||^{\left(m^{+}-n-1\right) / 2} \chi_{m} \otimes \pi\right)$ is reducible.

In the same way, using the theta lift $\theta_{\chi}\left(\tau^{-}, V_{n+2}\right)$, we deduce that the induced representation $\operatorname{Ind}_{P_{1}}^{\mathrm{U}\left(V_{n+2}\right)}\left(||^{\left(m^{-}-n-1\right) / 2} \chi_{m} \otimes \pi\right)$ is reducible. However [Sil, Lemma 1.2], implies that the representation

$$
\operatorname{Ind}_{P_{1}}^{\mathrm{U}\left(V_{n+2}\right)}\left(||^{s} \chi_{m} \otimes \pi\right)
$$

has at most just two real points of reducibility, of the form $s= \pm s_{0}$. If $m^{+} \neq m^{-}$we deduce that

$$
m^{+}-n-1=-\left(m^{-}-n-1\right),
$$

that is, $m^{+}+m^{-}=2 n+2$. If $m^{+}=m^{-}$, then by (1.1) and (1.2), the only possibility is $m^{+}=m^{-}=n+1$.

Remark 1.2. - We have, in fact, proved that the induced representation (1.3) is reducible exactly when $s \in\left\{\frac{m^{+}-n-1}{2}, \frac{m^{-}-n-1}{2}\right\}$. The reducibility points of the induced representation (1.3) may be computed in terms of $\pi$ (for example, by computing poles of L-functions when $\mathrm{F}$ is of zero characteristic and $\pi$ generic or with the theory of types if $n$ and $m$ are not too big). If so, we may deduce the set $\left\{m^{+}, m^{-}\right\}$. The set of points for which the representation (1.3) is reducible is supposed to be constant for representations in the same L-packet so we deduce that the set $\left\{m^{+}, m^{-}\right\}$is constant for cuspidal representations in the same L-packet.

For example, in [HKS, §7] it was expected that, if $m$ and $n$ are of different parity, then for most representations $\left\{m^{+}, m^{-}\right\}=\{n+1, n+1\}$ while, if $m$ and $n$ are of the 
same parity, then for most representations $\left\{m^{+}, m^{-}\right\}=\{n, n+2\}$. We can translate this fact now, for cuspidal representations, by saying that for most cuspidal representations $\pi,(1.3)$ is reducible for $s=0$ if $m$ and $n$ are of different parity, and for $s= \pm \frac{1}{2}$ if $m$ and $n$ are of the same parity.

\section{The dual pair $(\mathrm{O}(n), \mathrm{Sp}(2 m))$}

In this section, we consider the case of the orthogonal-symplectic dual pair. Let $V$ be a quadratic space over $\mathrm{F}$ of dimension $n$ and denote by $\mathrm{O}(V)$ the orthogonal group attached to it. For any positive integer $m$, let $W_{m}$ be a symplectic space over $\mathrm{F}$ of dimension $m$. Let $\mathrm{G}_{m}=\operatorname{Sp}\left(W_{m}\right)$ be the symplectic group of rank $m$ over $\mathrm{F}$, when $n$ is of even parity, or $\mathrm{G}_{m}=\mathrm{Mp}\left(W_{m}\right)$ be its metaplectic extension, in the case of odd parity. The groups $\mathrm{O}(V), \operatorname{Sp}\left(W_{m}\right)$ form a dual pair in $\operatorname{Sp}\left(V \otimes W_{m}\right)$. It splits when $n$ is even so we can consider the restriction of the metaplectic representation to the product $\mathrm{O}(V) \times \mathrm{G}_{m}$. For any irreducible cuspidal representation $\pi$ of $\mathrm{O}(V)$, denote $\theta\left(\pi, W_{m}\right)$ the theta lift of $\pi$ to $\mathrm{G}_{m}$, if such a lift exists. We write $\theta\left(\pi, W_{m}\right)=0$, if there is no such a lift.

The representation theory of $\mathrm{O}(V)$ follows from its connected component $\mathrm{SO}(V)$, see for example [MVW, 3.II.5]. Let sgn be the non trivial character of $\mathrm{O}(V) / \mathrm{SO}(V)$, and $\varepsilon_{n}$ an element of $\mathrm{O}(V)$ not in $\mathrm{SO}(V)$. For any irreducible representation $\pi_{0}$ of $\mathrm{SO}(V)$ we denote by $\pi_{0}^{\varepsilon_{n}}$ its conjugate by $\varepsilon_{n}$, that is

$$
\pi_{0}^{\varepsilon_{n}}\left(\varepsilon_{n} s \varepsilon_{n}^{-1}\right)=\pi_{0}(s), \quad s \in \mathrm{SO}(V) .
$$

The isomorphism class of $\pi_{0}^{\varepsilon_{n}}$ does not depend on the choice of $\varepsilon_{n}$.

Let $\pi$ be an irreducible cuspidal representation of $\mathrm{O}(V)$ and write $m^{+}$(resp. $m^{-}$) to be the smallest integer $m$ such that $\theta\left(\pi, W_{m}\right) \neq 0\left(\operatorname{resp} . \theta\left(\operatorname{sgn} \cdot \pi, W_{m}\right) \neq 0\right)$.

The following proposition, stating similar inequalities to (1.1) and (1.2), follows from the results of [Yam, §8], using the same arguments as in $[\mathbf{H K S}, \S 4]$ or $[\mathbf{K R}]$. For the sake of completeness, we will write a proof in the appendix.

Proposition 2.1. - The following inequalities hold:

$$
\begin{aligned}
& \max \left\{m^{+}, m^{-}\right\} \geq n / 2, \\
& \min \left\{m^{+}, m^{-}\right\} \leq n / 2 .
\end{aligned}
$$

Theorem 2.2. - We have

$$
m^{+}+m^{-}=n
$$


This theorem, for any irreducible representation, was conjectured in $[\mathbf{K R}$, Conjecture $\mathrm{C}$. Before proving the theorem, let's briefly recall some facts about the representation theory of orthogonal groups [MVW, 3.II.5]. Let $\pi$ be an irreducible representation of $\mathrm{O}(V)$ and let $\pi_{0}$ be an irreducible subrepresentation of the restriction of $\pi$ to $\mathrm{SO}(V)$. There are two different possibilities :

(1) The restriction $\pi$ to $\mathrm{SO}(V)$ is irreducible and hence equal to $\pi_{0}$. This is possible if and only if the following equivalent conditions are satisfied

(a) $\pi$ is not isomorphic to $\mathrm{sgn} \cdot \pi$;

(b) $\pi_{0}^{\varepsilon_{n}}$ is isomorphic to $\pi_{0}$;

(c) $\operatorname{Ind}_{\mathrm{SO}(V)}^{\mathrm{O}(V)}\left(\pi_{0}\right)=\pi+\operatorname{sgn} \cdot \pi$.

(2) The restriction $\pi$ to $\mathrm{SO}(V)$ is reducible and hence equal to $\pi_{0}+\pi_{0}^{\varepsilon_{n}}$. This is possible if and only if the following equivalent conditions are satisfied

(a) $\pi$ is isomorphic to $\operatorname{sgn} \cdot \pi$;

(b) $\pi_{0}^{\varepsilon_{n}}$ is not isomorphic to $\pi_{0}$;

(c) $\operatorname{Ind}_{\mathrm{SO}(V)}^{\mathrm{O}(V)}\left(\pi_{0}\right)=\pi$.

Define the quadratic space $V_{n+2}=V \oplus H$, where $H$ is a hyperbolic space. Denote by $\mathrm{P}_{1}$ (resp. $\left.\widetilde{\mathrm{P}}_{1}\right)$ the parabolic subgroup of $\mathrm{SO}\left(V_{n+2}\right)$ (resp. $\mathrm{O}\left(V_{n+2}\right)$ ) associated to this decomposition.

Proof. - Write $\tau^{+}=\theta\left(\pi, W_{m^{+}}\right)$and $\tau^{-}=\theta\left(\operatorname{sgn} \cdot \pi, W_{m^{-}}\right)$. It is known that $\tau^{ \pm}$is a cuspidal representation of $\mathrm{G}_{m^{ \pm}}$.

Consider the theta lift $\theta\left(\tau^{+}, V_{n+2}\right)$. By $[\mathbf{M V W}$, page 69] or [Ku1], the Jacquet functor $J_{P_{1}}\left(\theta\left(\tau^{+}, V_{n+2}\right)\right)$ is irreducible and $\theta\left(\tau^{+}, V_{n+2}\right)$ is a subquotient of the parabolically induced representation $\operatorname{Ind}_{\widetilde{\mathrm{P}}_{1}}^{\mathrm{O}\left(V_{n+2}\right)}\left(||^{\left(2 m^{+}-n\right) / 2} \otimes \pi\right)$. As the Jacquet functor of this induced representation, by the geometric lemma of [ $\mathbf{B Z}, 2.12]$, is reducible we deduce that $\operatorname{Ind}_{\widetilde{\mathrm{P}}_{1}}^{\mathrm{O}\left(V_{n+2}\right)}\left(\|^{\left(2 m^{+}-n\right) / 2} \otimes \pi\right)$ is reducible.

In the same way, using the theta lift $\theta\left(\tau^{-}, V_{n+2}\right)$, we deduce that the induced representation $\operatorname{Ind}_{\widetilde{\mathrm{P}}_{1}}^{\mathrm{O}\left(V_{n+2}\right)}\left(||^{\left(2 m^{-}-n\right) / 2} \otimes \operatorname{sgn} \cdot \pi\right)$ is reducible.

Suppose first that the restriction of $\pi$ to $\mathrm{SO}(V)$ is irreducible. Then,

$$
\begin{aligned}
\boldsymbol{r}_{\mathrm{SO}(V)}^{\mathrm{O}(V)}\left(\operatorname{Ind}_{\widetilde{\mathrm{P}}_{1}}^{\mathrm{O}\left(V_{n+2}\right)}\left(||^{\left(2 m^{+}-n\right) / 2} \otimes \pi\right)\right) & =\operatorname{Ind}_{\mathrm{P}_{1}\left(V_{n+2}\right)}^{\mathrm{SO}}\left(\|\left.\right|^{\left(2 m^{+}-n\right) / 2} \otimes \pi_{0}\right) \\
\boldsymbol{r}_{\mathrm{SO}(V)}^{\mathrm{O}(V)}\left(\operatorname{Ind}_{\widetilde{\mathrm{P}}_{1}}^{\mathrm{O}\left(V_{n+2}\right)}\left(||^{\left(2 m^{-}-n\right) / 2} \otimes \mathrm{sgn} \cdot \pi\right)\right) & =\operatorname{Ind}_{\mathrm{P}_{1}\left(V_{n+2}\right)}^{\mathrm{SO}\left(\|\left.\right|^{\left(2 m^{-}-n\right) / 2} \otimes \pi_{0}\right)},
\end{aligned}
$$


so the reducibility of the left-side representations implies the reducibility for the right-side representations. Again, [Sil, Lemma 1.2] implies that the representation $\operatorname{Ind}_{\widetilde{P}_{1}}^{\mathrm{SO}\left(V_{n+2}\right)}\left(||^{s} \otimes \pi_{0}\right)$ has at most just two real points of reducibility $s= \pm s_{0}$. If $m^{+} \neq m^{-}$, we deduce that

$$
2 m^{+}-n=-\left(2 m^{-}-n\right),
$$

that is, $m^{+}+m^{-}=n$.

If $m^{+}=m^{-}$, by Proposition 2.1, we get $m^{+}+m^{-}=n$. (See that this can just happen when $n$ is even.)

Suppose finally that the induced representation $\operatorname{Ind}_{\mathrm{SO}(V)}^{\mathrm{O}(V)}\left(\pi_{0}\right)$ is irreducible. This can happen just when $n$ is even : we have seen this means that $\pi$ is isomorphic to $\operatorname{sgn} \cdot \pi$ (and hence $m^{+}=m^{-}$) and $\pi_{0}$ is not isomorphic to $\pi_{0}^{\varepsilon_{n}}$. By Harish-Chandra criterion [Wa2, Prop IV.2.2], for any real $s$ the induced representation $\operatorname{Ind}_{\mathrm{P}_{1}}^{\mathrm{SO}\left(V_{n+2}\right)}\left(||^{s} \otimes \pi_{0}\right)$ is then irreducible.

This last representation, for $s \neq 0$, is not isomorphic to its conjugate by $\varepsilon_{n+2}$ so, as we have seen before, this means that the representation $\operatorname{Ind}_{\widetilde{\mathrm{P}}_{1}}^{\mathrm{O}\left(V_{n+2}\right)}\left(||^{s} \otimes \pi_{0}\right) \simeq$ $\operatorname{Ind}_{\mathrm{SO}(V)}^{\mathrm{O}(V)}\left(\operatorname{Ind}_{\mathrm{P}_{1}}^{\mathrm{SO}\left(V_{n+2}\right)}\left(||^{s} \otimes \pi_{0}\right)\right)$, is irreducible when $s \neq 0$. As it is reducible for $s=2 m^{+}-n$ we deduce that $2 m^{+}-n=0$ so $m^{+}=m^{-}=n / 2$ and hence $m^{+}+m^{-}=n$.

Remark 2.3. - We have, in fact, proved that the induced representation

$$
\operatorname{Ind}_{\widetilde{\mathrm{P}}_{1}}^{\mathrm{O}\left(V_{n+2}\right)}\left(||^{s} \otimes \pi_{0}\right)
$$

is reducible exactly when $s \in\left\{m^{+}-\frac{n}{2}, m^{-}-\frac{n}{2}\right\}$. The reducibility points of the induced representation (2.1) may be computed in terms of $\pi$ (for example with Shahidi's theory of L-functions when $\mathrm{F}$ is of zero characteristic and $\pi$ is generic). If so, we may deduce the set $\left\{m^{+}, m^{-}\right\}$.

There is a similar conjecture with the roles of $\mathrm{O}(V)$ and $\mathrm{G}_{m}$ exchanged. For a fixed quadratic character $\chi$ of $\mathrm{F}^{\times}$, there are precisely two isomorphism classes of quadratic spaces, $V_{n}^{ \pm}$, of dimension $n$, determined by the Hasse invariant. Let $\pi$ be a cuspidal representation of $\mathrm{G}_{m}$ and consider the collection of representations $\theta_{\chi}\left(\pi, V_{n}^{ \pm}\right)$as $n$ and \pm vary. Fix a parity of $n$ and define $n^{+}$(resp. $n^{-}$) to be the smallest integer $n$ with that fixed parity such that $\theta_{\chi}\left(\pi, V_{n}^{+}\right) \neq 0\left(\operatorname{resp} . \theta_{\chi}\left(\pi, V_{n}^{-}\right) \neq 0\right)$. 
Theorem 2.4. - We have

$$
n^{+}+n^{-}=4 m+4 .
$$

The proof is the same as for unitary groups (similar inequalities to (1.1) and (1.2) are proved in $[\mathbf{K R}]$ ) and is left to the reader. This theorem was first proved, with a different method, in $[\mathbf{K R}]$ for many irreducible representations (including cuspidal representations).

\section{Quaternionic dual pairs}

The literature about quaternionic dual pairs is not as large as for the other dual pairs. However, the proof of the conservation relation for cuspidal representations is much simpler in this case, as one does not need inequalities (1.1) and (1.2) to achieve it.

Let's first briefly recall the classification of quaternionic Hermitian and skew-Hermitian spaces. For a more detailed exposition one can consult [MVW], [GTn] and [Yam].

Let $\mathrm{F}$ be a non-Archimedean local field of characteristic not equal to 2 and let $\mathrm{D}$ be the quaternion $\mathrm{F}$-algebra. We denote by $\nu=|\mathrm{Nrd}|$ the normalized absolute value of the reduced norm of $\mathrm{D}$.

For a given positive integer $k$ there is a unique Hermitian space $V_{k}$ which has dimension $k$ over D. There are two Witt towers of quaternionic Hermitian spaces, characterized by the parity of their dimensions. We will denote by $\mathrm{U}\left(V_{k}\right)$ the automorphism group of $V_{k}$.

On the other hand for a given positive integer $k$ the quaternionic skew-Hermitian spaces of dimension $k$ over $\mathrm{D}$ are classified by their discriminant, which is an element in $\mathrm{F}^{\times} / \mathrm{F}^{\times 2}$ and thus determined by a quadratic character $\chi$ of $\mathrm{F}^{\times}$. Let fix a quadratic character $\chi$, and denote by $W_{k}$ the unique quaternionic skew-Hermitian spaces of dimension $k$ over $\mathrm{D}$ with discriminant $\chi$, except when $k=1$ and $\chi=1$ in which case $W_{k}$ does not exist. There are again two Witt towers of quaternionic skew-Hermitian spaces with fixed discriminant $\mathrm{K}$, characterized as before by the parity of their dimensions. We will denote by $\mathrm{U}\left(W_{k}\right)$ the automorphism group of $W_{k}$.

If $n$ and $m$ are positive integers, the groups $\mathrm{U}\left(V_{n}\right)$ and $\mathrm{U}\left(W_{m}\right)$ form a split reducive dual pair in $\operatorname{Sp}\left(V_{n} \otimes W_{m}\right)$. For any irreducible cuspidal representation $\pi$ of $\mathrm{U}\left(V_{n}\right)$, denote $\theta\left(\pi, W_{m}\right)$ the theta lift of $\pi$ to $\mathrm{U}\left(W_{m}\right)$, if such a lift exists. We write $\theta\left(\pi, W_{m}\right)=0$, if there is no such a lift.

Let us now fix the quaternionic Hermitian space $V_{n}$ and an irreducible cuspidal representation $\pi$ of $\mathrm{U}\left(V_{n}\right)$. Write $m^{+}$(resp. $m^{-}$) to be the smallest integer of the form $2 m$ 
(resp. $2 m+1)$ such that $\theta\left(\pi, W_{2 m}\right) \neq 0$ (resp. $\left.\theta\left(\pi, W_{2 m+1}\right) \neq 0\right)$. These are the first occurrence indices of $\pi$ in the two Witt towers.

Theorem 3.1. - We have

$$
m^{+}+m^{-}=2 n+3 .
$$

This equality was conjectured in [GTn, Conjecture 1] to hold for every irreducible representation $\pi$.

Proof. - Define the quaternionic Hermitian space $V_{n+2}=V_{n} \oplus H$, where $H$ is a hyperbolic space. Denote by $P_{1}$ the parabolic subgroup of $\mathrm{U}\left(V_{n+2}\right)$ associated to this decomposition. We will write $\tau^{+}=\theta\left(\pi, W_{m^{+}}\right)$and $\tau^{-}=\theta\left(\pi, V_{m^{-}}\right)$. It is known that $\tau^{ \pm}$is a cuspidal representation of $\mathrm{U}\left(W_{m^{ \pm}}\right)$.

Consider the theta lift $\theta\left(\tau^{+}, V_{n+2}\right)$. By [MVW, page 69], the Jacquet functor $J_{P_{1}}\left(\theta\left(\tau^{+}, V_{n+2}\right)\right)$ is irreducible and $\theta\left(\tau^{+}, V_{n+2}\right)$ is a subquotient of the parabolically induced representation $\operatorname{Ind}_{P_{1}}^{\mathrm{U}\left(V_{n+2}\right)}\left(\nu^{\left(m^{+}-n-\frac{3}{2}\right) / 2} \xi_{W_{m}}^{\psi} \otimes \pi\right)$, where $\xi_{W_{m}}^{\psi}$ is a certain fixed unitary character depending on the discriminant of $W_{m}$ and the choice of the non-trivial additive character $\psi$ of $\mathrm{F}$ (see Introduction). As the Jacquet functor of this induced representation, by the geometric lemma of $[\mathbf{B Z}, 2.12]$, is reducible we deduce that $\operatorname{Ind}_{P_{1}}^{\mathrm{U}\left(V_{n+2}\right)}\left(\nu^{\left(m^{+}-n-\frac{3}{2}\right) / 2} \xi_{W_{m}}^{\psi} \otimes \pi\right)$ is reducible.

In the same way, using the theta lift $\theta\left(\tau^{-}, V_{n+2}\right)$, we deduce that the induced representation $\operatorname{Ind}_{P_{1}}^{\mathrm{U}\left(V_{n+2}\right)}\left(\nu^{\left(m^{-}-n-\frac{3}{2}\right) / 2} \xi_{W_{m}}^{\psi} \otimes \pi\right)$ is reducible. However [Sil, Lemma 1.2] implies that the representation

$$
\operatorname{Ind}_{P_{1}}^{\mathrm{U}\left(V_{n+2}\right)}\left(\nu^{s} \xi_{W_{m}}^{\psi}, \otimes \pi\right)
$$

has just two real points of reducibility $s= \pm s_{0}$. As $m^{+} \neq m^{-}$(being of different parity) we deduce that

$$
m^{+}-n-\frac{3}{2}=-\left(m^{-}-n-\frac{3}{2}\right)
$$

that is, $m^{+}+m^{-}=2 n+3$.

Remark 3.2. - We have proved that the induced representation (3.1) is reducible exactly when $s \in\left\{\frac{m^{+}-n-3 / 2}{2}, \frac{m^{-}-n-3 / 2}{2}\right\}$. So similar arguments as in Remarks 1.2 and 2.3 apply.

One also has an analogous theorem with the role of $V_{n}$ and $W_{m}$ exchanged. Fix the skew-quaternionic Hermitian space $W_{m}$ and an irreducible cuspidal representation $\pi$ of $\mathrm{U}\left(W_{m}\right)$. Write $n^{+}$(resp. $n^{-}$) to be the smallest integer of the form $2 n$ (resp. $2 n+1$ ) such 
that $\theta\left(\pi, V_{2 n}\right) \neq 0\left(\right.$ resp. $\left.\theta\left(\pi, V_{2 n+1}\right) \neq 0\right)$. These are the first occurrence indices of $\pi$ in the two Witt towers.

Theorem 3.3. - We have

$$
n^{+}+n^{-}=2 m+1
$$

This equality was conjectured in [GTn, Conjecture 2] to hold for every irreducible representation $\pi$. The proof is the same (one just needs to change $\frac{3}{2}$ into $\frac{1}{2}$ in the exponents, as $W_{m}$ is now skew-Hermitian) and it is left to the reader.

A

\section{Proof of Proposition 2.1}

We keep notations as in Section 2. Let fix $V$ be a quadratic space over $\mathrm{F}$ of dimension $n$ and, for any positive integer $m$, let $W_{m}$ be a symplectic space over $\mathrm{F}$ of dimension $m$. The following facts are well known so we will not give many details.

We denote by $R_{n}\left(W_{m}\right)$ the maximal quotient of the space of Bruhat functions $\mathscr{S}\left(W_{m}^{n}\right)$ on which $\operatorname{Sp}\left(W_{m}\right)$ acts trivially. Then, if we denote by $\mathrm{O}(n, n)$ the split orthogonal group of rank $2 n$, by $[\mathbf{R a l}], R_{n}\left(W_{m}\right)$ injects into the degenerate principal series

$$
\operatorname{Ind}_{\mathrm{GL}_{n}(\mathrm{~F}) \times \mathrm{GL}_{n}(\mathrm{~F})}^{\mathrm{O}(n, n)}\left(|\operatorname{det}|^{m-\frac{n-1}{2}}\right) .
$$

The interest of using $R_{n}\left(W_{m}\right)$ comes from the fact that (A.1) is a well known representation (in our case, this work has been achieved by Yamana [Yam, §8]) and the fundamental equivalence (see [Ku2, §IV, Prop. 1.10], for example)

$$
\theta\left(\pi, W_{m}\right) \neq 0 \Leftrightarrow \operatorname{Hom}_{\mathrm{O}(V) \times \mathrm{O}(V)}\left(R_{n}\left(W_{m}\right), \pi \otimes \pi\right) \neq 0 .
$$

Let fix now $\pi$ a cuspidal representation of $\mathrm{O}(V)$. By [MVW, page71],

$$
\operatorname{dim}\left(\operatorname{Hom}_{\mathrm{O}(V) \times \mathrm{O}(V)}\left(\operatorname{Ind}_{\mathrm{GL}_{n}(\mathrm{~F}) \times \mathrm{GL}_{n}(\mathrm{~F})}^{\mathrm{O}(n, n)}\left(|\operatorname{det}|^{m-\frac{n-1}{2}}\right), \pi \otimes \pi\right)\right)=1 .
$$

Suppose that $m<\frac{n}{2}$. By [Yam, Proposition 8.4], we deduce from the previous equality that,

$\operatorname{dim}\left(\operatorname{Hom}_{\mathrm{O}(V) \times \mathrm{O}(V)}\left(\left(R_{n}\left(W_{m}\right), \pi \otimes \pi\right)\right)+\operatorname{Hom}_{\mathrm{O}(V) \times \mathrm{O}(V)}\left(\left(R_{n}\left(W_{m}\right), \operatorname{sgn} \cdot \pi \otimes \operatorname{sgn} \cdot \pi\right)\right)\right) \leq 1$, 
so, by (A.2), we cannot have that both $\theta\left(\pi, W_{m}\right)$ and $\theta\left(\operatorname{sgn} \cdot \pi, W_{m}\right)$ are non zero. By the persistence principle [MVW, page 67], this exactly means that

$$
\max \left\{m^{+}, m^{-}\right\} \geq \frac{n}{2}
$$

On the other hand, if $m \geq \frac{n}{2}$, by [Yam, Corollary 8.8] and (A.3), $\operatorname{dim}\left(\operatorname{Hom}_{\mathrm{O}(V) \times \mathrm{O}(V)}\left(\left(R_{n}\left(W_{m}\right), \pi \otimes \pi\right)\right)+\operatorname{Hom}_{\mathrm{O}(V) \times \mathrm{O}(V)}\left(\left(R_{n}\left(W_{m}\right), \operatorname{sgn} \cdot \pi \otimes \operatorname{sgn} \cdot \pi\right)\right)\right) \geq 1$, so at least one of the representations $\theta\left(\pi, W_{m}\right)$ or $\theta\left(\operatorname{sgn} \cdot \pi, W_{m}\right)$ is not zero. Again, by the persistence principle [MVW, page 67], this exactly means that

$$
\min \left\{m^{+}, m^{-}\right\} \leq \frac{n}{2}
$$

which proves the proposition.

Acknowledgments. - I would like to thank Dipendra Prasad for his kind hospitality during my visit to the Tata Insitute of Bombay when this article was first written. I would like to thank as well C. Jantzen and C. Moglin for their useful comments about the representation theory of orthogonal groups, and the referee for useful remarks.

\section{References}

[BZ] I.N. Bernstein, A.V. Zelevinskii, Induced Representations of Reductive p-Adic Groups I, Ann. Scient. Ec. Norm. Sup., $4^{\mathrm{e}}$ serie, t. 10, 1977, 441-472.

[GTk] W. T. Gan, S. Takeda, The local Langlands conjecture for GSp(4), preprint 2009, to appear in Annals of Math.

[GTn] W. T. Gan, W. Tantono, The local Langlands conjecture for GSp(4) II : the case of inner forms, preprint 2009.

[GG] Z. Gong, L. Grenié, An inequality for local Theta correspondence, preprint 2009, arXiv : 0706.0952.

[Han] M. Hanzer Rank one reducibility for unitary groups, preprint 2010.

[Ku1] S. S. Kudla, On the local theta correspondence, Inventiones math. 83 (1986), 229-255.

[Ku2] S. S. Kudla, Notes on the local theta correspondence, Preprint 1996, available at http://www.math.toronto.edu/ skudla/castle.pdf

$[\mathrm{KR}] \quad$ S. S. Kudla, S. Rallis, On the first ocurrence in the local theta correspondence, in Automorphic representations, $L$-functions and applications: progress and prospects, vol. 11 of Ohio state Univ. Math. Res. Inst. Publ., de Gruyter, Berlin, 2005, pp. 273-308.

[HKS] M. Harris, S. S. Kudla, J. Sweet, Theta dichotomy for unitary groups, Journal of the AMS, vol. 9, pp. 941-1004 (1996).

[MVW] C. Møglin, M.F. Vignéras, J.L. Waldspurger, Correspondance de Howe sur un corps p-adique, LNM 1291, Springer-Verlag, 1987. 
[Sil] A. J. Silberger, Special representations of reductive p-adic groups are not integrable, Ann. of Math. (2), 111 (1980), pp. 571-587.

[Ral] S. Rallis, On the Howe duality conjecture, Compos. Math 51, 333-399 (1984).

[Wa1] J.L. Waldspurger, Démonstration d'une conjecture de dualité de Howe dans le cas padique, $p \neq 2$, in: Festschrift in honor of Piatetski-Shapiro, Israel Math. Conf. Proc., vol 2, 1990, 267-324.

[Wa2] J.L. Waldspurger, La formule de Plancherel pour les groupes p-adiques, d'après HarishChandra, J. of the Inst. of Math. Jussieu (2003) 2 (2), 235-333.

[Yam] S. Yamana, Degenerate principal series representations for quaternionic unitary groups, preprint 2010, to appear in Israel Journal of Math.

Alberto Mínguez, Institut de Mathématiques de Jussieu, Université Paris 6. 175, rue de Chevaleret. 75013 Paris, France., URL: http://www.institut.math.jussieu.fr/ minguez/

E-mail : minguez@math.jussieu.fr 